\title{
CHARACTERIZATION OF THE STABILITY SET FOR NON-DIFFERENTIABLE FUZZY PARAMETRIC OPTIMIZATION PROBLEMS
}

\author{
MOHAMED ABD EL-HADY KASSEM
}

Received 3 November 2003

This note presents the characterization of the stability set of the first kind for multiobjective nonlinear programming (MONLP) problems with fuzzy parameters either in the constraints or in the objective functions without any differentiability assumptions. These fuzzy parameters are characterized by triangular fuzzy numbers (TFNs). The existing results concerning the parametric space in convex programs are reformulated to study for multiobjective nonlinear programs under the concept of $\alpha$-Pareto optimality.

\section{Introduction}

In an earlier work, Mangasarian [6] introduced the Kuhn-Tucker saddle point (KTSP) necessary and sufficiency optimality theorems for nonlinear programming problems. Tanaka and Asai [10] formulated multiobjective linear programming problems with fuzzy parameters. Sakawa and Yano [9] introduced the concept of $\alpha$-Pareto optimality of fuzzy parametric programs. Orlovski [7] formulated general multiobjective nonlinear programming problems with fuzzy parameters. Kaufmann and Gupta [5] introduced the concept of triangular fuzzy numbers (TFNs). Osman and El-Banna [8] introduced the stability of multiobjective nonlinear programming problems with fuzzy parameters. Kassem [2,3] introduced an algorithm for multiobjective nonlinear programming problems with fuzzy parameters in the constraints and determined the stability set of the first kind for these problems, also introduced a method for decomposing the fuzzy parametric space in multiobjective nonlinear programming problems using the generalized Tchebycheff norm (GTN).

This note gives the characterization of the stability set of the first kind for convex multiobjective nonlinear programming (MONLP) problems with fuzzy parameters in the constraints and for convex MONLP problems with fuzzy parameters in the objective functions. These fuzzy parameters are characterized by TFNs and treatment under the concept of $\alpha$-Pareto optimality. In this note, no differentiability assumptions are needed and the KTSP is used in the derivation of the proposed results. 


\section{Problem formulation}

We consider the following convex MONLP problem with fuzzy parameters in the righthand side of constraints:

$$
\min \left(f_{1}(x), \ldots, f_{m}(x)\right)
$$

subject to

$$
x \in X(\widetilde{v})=\left\{x \in \mathbb{R}^{n}: g_{j}(x) \leq \tilde{v_{j}}, j=1,2, \ldots, k\right\},
$$

where the functions $f_{i}(x), i=1,2, \ldots, m$, and $g_{j}(x), j=1,2, \ldots, k$ are assumed to be convex on $\mathbb{R}^{n}$ and $\tilde{v_{j}}, j=1,2, \ldots, k$, are any real fuzzy parameters which are characterized by real fuzzy numbers that form a convex continuous fuzzy subset of the real line whose membership functions are $\mu_{\tilde{v}_{j}}\left(v_{j}\right), j=1,2, \ldots, k$. There is an infinite set of fuzzy numbers, but here we will define a special class of fuzzy numbers called TFNs which can be defined by a triplet $\left(v^{1}, v^{2}, v^{3}\right)$, that is, the membership functions $\mu_{v_{j}}\left(v_{j}\right), j=1,2, \ldots, k$, are functions of $v^{t}, t=1,2,3$. These membership functions $\mu_{\widetilde{v}_{j}}\left(v_{j}\right), j=1,2, \ldots, k$, are defined by [5]

$$
\mu_{\widetilde{v}_{j}}\left(v_{j}\right)= \begin{cases}0, & v_{j} \leq v_{j}^{1}, \\ \frac{v_{j}-v_{j}^{1}}{v_{j}^{2}-v_{j}^{1}}, & v_{j}^{1} \leq v_{j} \leq v_{j}^{2}, \\ \frac{v_{j}^{3}-v_{j}}{v_{j}^{3}-v_{j}^{2}}, & v_{j}^{2} \leq v_{j} \leq v_{j}^{3}, \\ 0, & v_{j} \geq v_{j}^{3},\end{cases}
$$

which are nondifferentiable on $\left[v_{j}^{1}, v_{j}^{3}\right], j=1,2, \ldots, k$.

Definition 2.1. The $\alpha$-level set of the fuzzy numbers $\tilde{v}_{j}, j=1,2, \ldots, k$, is defined as the ordinary set $L_{\alpha}(\widetilde{v})$ for which the degree of their membership functions exceeds the level $\alpha[1]:$

$$
L_{\alpha}(\widetilde{v})=\left\{v: \mu_{\widetilde{v}_{j}}\left(v_{j}\right) \geq \alpha, j=1,2, \ldots, k\right\}
$$

For a certain degree of $\alpha$, the MONLP problem (2.1)-(2.2) can be written in the following nonfuzzy form [9]:

$$
\min \left(f_{1}(x), \ldots, f_{m}(x)\right)
$$

subject to

$$
N(v)=\left\{(x, v) \in \mathbb{R}^{n+k}: g_{j}(x) \leq v_{j}, j=1,2, \ldots, k ; \mu_{v_{j}}\left(v_{j}\right) \geq \alpha, j=1,2, \ldots, k\right\} .
$$

The scalarization form for the problem (2.5)-(2.6) is

$$
\min \sum_{i=1}^{m} w_{i} f_{i}(x)
$$


subject to

$$
(x, v) \in N(v),
$$

where $w_{i} \geq 0, i=1,2, \ldots, m$, for at least one $i$ satisfying $w_{i}>0$ and $\sum_{i=1}^{m} w_{i}=1$.

Definition 2.2. For a certain degree of $\alpha$, suppose that the problem (2.7)-(2.8) is solvable for $(w, v)=(\bar{w}, \bar{v})$ with an $\alpha$-optimal point $(\bar{x}, \bar{v})$, then the $\alpha$-stability set of the first kind of problem (2.7)-(2.8) corresponding to $(\bar{x}, \bar{v})$ denoted by $S(\bar{x}, \bar{v})$ is defined by

$$
S(\bar{x}, \bar{v})=\left\{\left(w, v^{t}\right) \in \mathbb{R}^{m+3 k}: \sum_{i=1}^{m} w_{i} f_{i}(\bar{x})=\min _{(x, v) \in N(v)} \sum_{i=1}^{m} w_{i} f_{i}(x)\right\}
$$

where $\mathbb{R}^{m}$ is the $m$-dimensional vector space of weights and $\mathbb{R}^{3 k}$ is the $3 k$-dimensional vector space of fuzzy parameters which are characterized by TFNs.

Lemma 2.3. For a certain degree of $\alpha$, if the problem (2.7)-(2.8) is stable for all $\left(w, v^{t}\right)$ such that $N(v) \neq \phi$, then $g(\bar{x})=\bar{v}, \mu_{\widetilde{v}}\left(\bar{v}^{t}\right)=\alpha,\left(\bar{w}, \bar{v}^{t}\right) \in S(\bar{x}, \bar{v})$.

Proof. If $S(\bar{x}, \bar{v})=\left\{\left(\bar{w}, \bar{v}^{t}\right)\right\}$, then the result is clear. Assume that $\left(\widehat{w}, \hat{v}^{t}\right) \in S(\bar{x}, \bar{v}),\left(\bar{w}, \bar{v}^{t}\right) \neq$ $\left(\widehat{w}, \hat{v}^{t}\right)$, then by the assumption and from the KTSP necessary optimality theorem [6], it follows that $(\bar{x}, \bar{v})$ and some $\hat{u} \geq 0, \hat{\eta} \geq 0$ solve $\operatorname{KTSP}$ and $\widehat{u}_{j}\left(g_{j}(\bar{x})-\bar{v}_{j}\right)=0, \hat{\eta}_{r}(\alpha-$ $\left.\mu_{\hat{v}_{r}}\left(\hat{v}_{r}^{t}\right)\right)=0$, that is, for $\alpha=\bar{\alpha}$, we have

$$
\tau\left(\bar{x}, \bar{w}, \bar{v}, u, \eta, \hat{v}^{t}\right) \leq \tau\left(\bar{x}, \bar{w}, \bar{v}, \hat{u}, \hat{\eta}, \hat{v}^{t}\right) \leq \tau\left(x, w, v, \hat{u}, \hat{\eta}, \hat{v}^{t}\right)
$$

for all $(x, v) \in N(v)$ and for all $u \geq 0, \eta \geq 0$ and $\hat{u}[g(\bar{x})-\bar{v}]=0, \hat{\eta}\left[\bar{\alpha}-\mu_{\widetilde{v}}\left(\hat{v}^{t}\right)\right]=0$, where

$$
\tau\left(x, w, v, u, \eta, v^{t}\right)=\sum_{i=1}^{m} w_{i} f_{i}(x)+\sum_{j=1}^{k} u_{j}\left[g_{j}(x)-v_{j}\right]-\sum_{r=1}^{k} \eta_{r}\left[\mu_{\widetilde{v}_{r}}\left(v_{r}^{t}\right)-\alpha\right]
$$

since $\hat{u}_{j} g_{j}(\bar{x})=\hat{u}_{j} \bar{v}_{j}, j=1,2, \ldots, k$, and $\hat{\eta}_{r} \mu_{v_{r}}\left(\widehat{v}_{r}^{t}\right)=\hat{\eta}_{r} \bar{\alpha}$. Then

$$
\tau\left(\bar{x}, \bar{w}, \bar{v}, u, \eta, \hat{v}^{t}\right) \leq \sum_{i=1}^{m} \overline{w_{i}} f_{i}(\bar{x}) \leq \tau\left(x, w, v, \hat{u}, \hat{\eta}, \hat{v}^{t}\right)
$$

from which it follows that

$$
\tau\left(\bar{x}, \bar{w}, \bar{v}, u, \eta, \bar{v}^{t}\right) \leq \tau\left(\bar{x}, \bar{w}, \bar{v}, \hat{u}, \hat{\eta}, \bar{v}^{t}\right) \leq \tau\left(x, w, v, \hat{u}, \hat{\eta}, \bar{v}^{t}\right)
$$

Therefore, from the KTSP sufficient optimality theorem, it follows that $g(\bar{x})=\bar{v}$, $\mu_{\widetilde{v}}\left(\bar{v}^{t}\right)=\bar{\alpha}$, that is, $\left(\bar{w}, \bar{v}^{t}\right) \in S(\bar{x}, \bar{v})$.

Theorem 2.4. For a certain degree of $\alpha$, if the problem (2.7)-(2.8) is stable for all $\left(w, v^{t}\right)$ such that $N(v) \neq \phi$, then the set $S(\bar{x}, \bar{v})$ is star shaped with point of common visibility $\bar{v}=g(\bar{x})$, $\bar{\alpha}=\mu_{\tilde{v}}\left(\widetilde{v}^{t}\right)$ and closed. 
Proof. From the previous lemma, it follows that $\left(\bar{w}, \bar{v}^{t}\right) \in S(\bar{x}, \bar{v})$. Let other point $\left(\widetilde{w}, \widetilde{v}^{t}\right) \in$ $S(\bar{x}, \bar{v})$, then by the assumption and from the KTSP necessary optimality theorem, it follows that $(\bar{x}, \bar{v})$ and some $\tilde{u} \geq 0, \tilde{\eta} \geq 0$ solve $\operatorname{KTSP}[6]$ and $\tilde{u}[g(\bar{x})-\bar{v}]=0, \tilde{\eta}\left[\bar{\alpha}-\mu_{\widetilde{v}}\left(\bar{v}^{t}\right)\right]=$ 0 , then for all $(x, v) \in \mathbb{R}^{n+k}, u \geq 0, \eta \geq 0$, we have

$$
\tau\left(\bar{x}, \widetilde{w}, \bar{v}, u, \eta, \widetilde{v}^{t}\right) \leq \tau\left(\bar{x}, \tilde{w}, \bar{v}, \tilde{u}, \tilde{\eta}, \widetilde{v}^{t}\right) \leq \tau\left(x, w, v, \tilde{u}, \tilde{\eta}, \widetilde{v}^{t}\right)
$$

and $\tilde{u}_{j}\left[g_{j}(\bar{x})-\bar{v}_{j}\right]=0, \tilde{\eta}_{r}\left[\bar{\alpha}-\mu_{\widetilde{v}}\left(\widetilde{v}^{t}\right)\right]=0$.

Putting $u_{j}=\gamma U_{j}, \zeta_{r}=\gamma \eta_{r}, w_{i}=\gamma W_{i}, \gamma \geq 0$, and $(1-\gamma) \tilde{u}_{j}\left[g_{j}(\bar{x})-\bar{v}_{j}\right]=0,(1-\gamma) \tilde{\eta}_{r}[\bar{\alpha}-$ $\left.\mu \widetilde{v}\left(\tilde{v}^{t}\right)\right]=0$.

For a certain degree of $\alpha=\bar{\alpha}$, we deduce from the relation (2.14) that

$$
\begin{aligned}
& \sum_{i=1}^{m} \tilde{w}_{i} f_{i}(\bar{x})+\sum_{j=1}^{k} u_{j}\left[g_{j}(\bar{x})-\bar{v}_{j}\right]-\sum_{r=1}^{k} \eta_{r}\left[\mu \widetilde{v}_{r}\left(\widetilde{v}^{t}\right)-\bar{\alpha}\right] \\
& \quad \leq \sum_{i=1}^{m} \tilde{w}_{i} f_{i}(\bar{x})+\sum_{j=1}^{k} \tilde{u}_{j}\left[g_{j}(\bar{x})-\bar{v}_{j}\right]-\sum_{r=1}^{k} \tilde{\eta}_{r}\left[\mu \widetilde{v}_{r}\left(\widetilde{v}_{r}^{t}\right)-\bar{\alpha}\right] \\
& \quad \leq \sum_{i=1}^{m} w_{i} f_{i}(x)+\sum_{j=1}^{k} \tilde{u}_{j}\left[g_{j}(x)-\bar{v}_{j}\right]-\sum_{r=1}^{k} \tilde{\eta}_{r}\left[\mu \widetilde{v}_{r}\left(\widetilde{v}_{r}^{t}\right)-\bar{\alpha}\right] .
\end{aligned}
$$

That is, for all $U \geq 0, \zeta \geq 0$, and $\gamma \geq 0$, we have

$$
\begin{aligned}
\sum_{i=1}^{m} \tilde{w}_{i}[ & \left.f_{i}(\bar{x})-(1-\gamma) f_{i}(\bar{x})\right]+\sum_{j=1}^{k} U_{j}\left[g_{j}(\bar{x})-(1-\gamma) g_{j}(\bar{x})-\gamma \bar{v}_{j}\right] \\
& -\sum_{r=1}^{k} \zeta_{r}\left[\mu_{\widetilde{v}_{r}}\left(\widetilde{v}^{t}\right)-\alpha-(1-\gamma)\left(\mu_{\widetilde{v}}\left(\widetilde{v}^{t}\right)-\alpha\right)\right] \\
\leq & \sum_{i=1}^{m} \widetilde{w}_{i} f_{i}(\bar{x})+\sum_{j=1}^{k} \tilde{u}_{j}\left[g_{j}(\bar{x})-\bar{v}_{j}\right]-\sum_{r=1}^{k} \tilde{\eta}_{r}\left[\mu_{\widetilde{v}_{r}}\left(\widetilde{v}^{t}\right)-\alpha\right] \\
\leq & \sum_{i=1}^{m} W_{i}\left[f_{i}(x)-(1-\gamma) f_{i}(x)\right]+\sum_{j=1}^{k} \tilde{U}_{j}\left[g_{j}(x)-(1-\gamma) g_{j}(\bar{x})-\gamma v_{j}\right] \\
& -\sum_{r=1}^{k} \widetilde{\zeta}_{r}\left[\left(\mu_{\widetilde{v}_{r}}\left(\tilde{v}_{r}^{t}\right)-\alpha\right)-(1-\gamma)\left(\mu_{v_{r}}\left(\tilde{v}^{t}\right)-\alpha\right)\right] .
\end{aligned}
$$

Therefore, it follows from the KTSP sufficient optimality theorem and from the relation (2.16) that $(1-\gamma) w_{i} f_{i}(\bar{x}),(1-\gamma) g_{j}(\bar{x})+\gamma \bar{v}$, and $\gamma \bar{\alpha}-(1-\gamma) \mu_{\widetilde{v}}\left(\widetilde{v}^{t}\right)$ belong to $S(\bar{x}, \bar{v})$ for all $\gamma \geq 0$. Hence the set $S(\bar{x}, \bar{v})$ is star shaped with point of common visibility $\left(\bar{w}, \bar{v}^{t}\right)$.

If the set $S(\bar{x}, \bar{v})$ is a one-point set or the whole space, then it is clearly closed. Choose a sequence of points $\left(w^{(n)}, v^{t_{n}}\right) \in S(\bar{x}, \bar{v})$ which is convergent to $\left(w, v^{t}\right)$, that is, $\lim _{n \rightarrow \infty}\left(w^{(n)}\right.$, $\left.v^{t_{n}}\right)=\left(w, v^{t}\right)$. Then $\sum_{i=1}^{m} w_{i}^{(n)} f_{i}(\bar{x}) \leq \sum_{i=1}^{m} w_{i}^{(n)} f_{i}(x)$ for all $g(x) \leq v^{t_{n}}, \mu_{\widetilde{v}}\left(v^{t_{n}}\right) \geq \alpha, n=1$, $2, \ldots$. Taking the limit as $n \rightarrow \infty$, we get for all $g(x) \leq v^{t}, \mu_{\tilde{v}}\left(v^{t}\right) \geq \alpha$ that

$$
\sum_{i=1}^{m} w_{i} f_{i}(\bar{x}) \leq \sum_{i=1}^{m} w_{i} f_{i}(x)
$$

that is, $\left(w, v^{t}\right) \in S(\bar{x}, \bar{v})$, and therefore the set $S(\bar{x}, \bar{v})$ is closed.

Theorem 2.5. For a certain degree of $\alpha$, if the problem (2.7)-(2.8) is stable for all $\left(w, v^{t}\right)$ such that $N(v) \neq \phi$, then the set $S(\bar{x}, \bar{v})$ is either one-point set or unbounded. 
Proof. If the set $S(\bar{x}, \bar{v})$ consists only of one point, then it is clear that this point is $\bar{v}_{j}=$ $g_{j}(\bar{x}), \mu_{\widetilde{v}_{j}}\left(\widetilde{v}_{j}^{t}\right) \geq \alpha$. If $S(\bar{x}, \bar{v})$ contains another point $\left(w^{\prime}, v^{t^{\prime}}\right)$, then it is clear from $(2.16)$ and from the KTSP sufficient optimality theorem that

$$
\left\{\left(w, v^{t}\right) \in \mathbb{R}^{m+3 k}:(1-\gamma) w^{\prime} f(\bar{x}),(1-\gamma) g(\bar{x})+\gamma v^{\prime},(1-\gamma) \mu_{v}\left(v^{t}\right)-\gamma \alpha\right\} \subseteq S(\bar{x}, \bar{v}), \quad \gamma \geq 0,
$$

that is, implying that the set $S(\bar{x}, \bar{v})$ is unbounded.

\section{Fuzzy parameters in the objective functions}

We consider the following convex MONLP problem with fuzzy parameters in the objective functions:

$$
\min \left(f_{1}\left(x, \tilde{\lambda}_{1}\right), \ldots, f_{m}\left(x, \tilde{\lambda}_{m}\right)\right)
$$

subject to

$$
x \in X=\left\{x \in \mathbb{R}^{n}: g_{j}(x) \leq 0, j=1,2, \ldots, k\right\},
$$

where the functions $f_{i}\left(x, \lambda_{i}\right), i=1,2, \ldots, m$, and $g_{j}(x), j=1,2, \ldots, k$, are assumed to be convex on $\mathbb{R}^{n}$ and $\tilde{\lambda} \in \mathbb{R}^{m}$ is the $m$-dimensional vector space of fuzzy parameters which are characterized by TFNs.

The corresponding scalarization problem with fuzzy parameters is

$$
\min \sum_{i=1}^{m} w_{i} f_{i}\left(x, \tilde{\lambda}_{i}\right)
$$

subject to

$$
x \in X
$$

where $w_{i} \geq 0, i=1,2, \ldots, m$, for at least one $i$ satisfying $w_{i}>0$ and $\sum_{i=1}^{m} w_{i=1}=1$.

For a certain degree of $\alpha$, the above problem can be written in the following nonfuzzy form [9]:

$$
\min F(x, w, \lambda)=\sum_{i=1}^{m} w_{i} f_{i}\left(x, \lambda_{i}\right)
$$

subject to

$$
M(\lambda)=\left\{(x, \lambda) \in \mathbb{R}^{n+m}: g_{j}(x) \leq 0, j=1,2, \ldots, k ; \mu_{\tilde{\lambda}_{i}}\left(\lambda_{i}\right) \geq \alpha, i=1,2, \ldots, m\right\},
$$

where $\mu_{\tilde{\lambda}_{i}}\left(\lambda_{i}\right), i=1,2, \ldots, m$, are defined as the previous form of $\mu_{\widetilde{v}_{j}}\left(v_{j}\right)$, which are nondifferentiable on $\left[\lambda_{i}^{1}, \lambda_{i}^{3}\right]$.

Definition 3.1. For a certain degree of $\alpha$, suppose that the problem (3.5)-(3.6) is solvable for $(w, \lambda)=(\bar{w}, \bar{\lambda})$ with an $\alpha$-optimal point $(\bar{x}, \bar{\lambda})$, then the $\alpha$-stability set of the first kind of problem (3.5)-(3.6) corresponding to $(\bar{x}, \bar{\lambda})$ denoted by $T(\bar{x}, \bar{\lambda})$ is defined by

$$
T(\bar{x}, \bar{\lambda})=\left\{\left(w, \lambda^{t}\right) \in \mathbb{R}^{4 m}: \sum_{i=1}^{m} w_{i} f_{i}\left(\bar{x}, \overline{\lambda_{i}}\right)=\min _{(x, \lambda) \in M(\lambda)} \sum_{i=1}^{m} w_{i} f_{i}\left(x, \lambda_{i}\right)\right\},
$$


where $\mathbb{R}^{4 m}=\mathbb{R}^{m+3 m}, \mathbb{R}^{m}$ is the $m$-dimensional vector space of weights, and $\mathbb{R}^{3 m}$ is the $3 m$-dimensional vector space of fuzzy parameters which are characterized by the TFNs.

Theorem 3.2. For a certain degree of $\alpha$, the set $T(\bar{x}, \bar{\lambda})$ is convex and closed.

Proof. For a certain degree of $\alpha$, if the set $T(\bar{x}, \bar{\lambda})$ is a one-point set or the whole space, it is clearly convex and closed. Suppose that $\left(w^{1}, \lambda^{t_{1}}\right)$ and $\left(w^{2}, \lambda^{t_{2}}\right)$ are any two points in $T(\bar{x}, \bar{\lambda})$, then for all $(x, \lambda) \in M(\lambda)$, we have

$$
\begin{aligned}
& \tau\left(\bar{x}, \bar{w}^{1}, \bar{\lambda}, u, \eta, \bar{\lambda}^{t_{1}}\right) \leq \tau\left(\bar{x}, \bar{w}^{1}, \bar{\lambda}, \hat{u}, \hat{\eta}, \tilde{\lambda}^{t_{1}}\right) \leq \tau\left(x, w^{1}, \lambda, \hat{u}, \hat{\eta}, \tilde{\lambda}^{t_{1}}\right), \\
& \tau\left(\bar{x}, \bar{w}^{2}, \bar{\lambda}, u, \eta, \bar{\lambda}^{t_{2}}\right) \leq \tau\left(\bar{x}, \bar{w}^{2}, \bar{\lambda}, \hat{u}, \hat{\eta}, \tilde{\lambda}^{t_{2}}\right) \leq \tau\left(x, w^{2}, \lambda, \hat{u}, \hat{\eta}, \tilde{\lambda}^{t_{2}}\right),
\end{aligned}
$$

where

$$
\tau\left(x, w, \lambda, u, \eta, \lambda^{t}\right)=\sum_{i=1}^{m} w_{i} f_{i}\left(x, \lambda_{i}\right)+\sum_{j=1}^{k} u_{j} g_{j}(x)-\sum_{r=1}^{m} \eta_{r}\left(\mu_{\tilde{\lambda}_{r}}\left(\lambda_{r}\right)-\alpha\right) .
$$

Therefore,

$$
\tau\left(\bar{x}, \widehat{w}, \bar{\lambda}, u, \eta, \hat{\lambda}^{t}\right) \leq \tau\left(\bar{x}, \widehat{w}, \bar{\lambda}, \hat{u}, \hat{\eta}, \hat{\lambda}^{t}\right) \leq \tau\left(x, \widehat{w}, \lambda, \hat{u}, \hat{\eta}, \hat{\lambda}^{t}\right)
$$

where $\hat{w}=(1-\gamma) \bar{w}^{1}+\gamma \bar{w}^{2}, \hat{\lambda}^{t}=(1-\gamma) \tilde{\lambda}^{t_{1}}+\gamma \tilde{\lambda}^{t_{2}}, 0 \leq \gamma \leq 1$, that is, $\left(\hat{w}, \hat{\lambda}^{t}\right) \in T(\bar{x}, \bar{\lambda})$, hence the set $T(\bar{x}, \bar{\lambda})$ is convex.

We choose a sequence of points $\left(w^{(n)}, \lambda^{t_{n}}\right) \in T(\bar{x}, \bar{\lambda})$ which is convergent to $\left(\bar{w}, \bar{\lambda}^{t}\right)$, then

$$
\tau\left(\bar{x}, w^{(n)}, \bar{\lambda}, u, \eta, \lambda^{t_{n}}\right) \leq \tau\left(x, w^{(n)}, \lambda, \hat{u}, \hat{\eta}, \lambda^{t_{n}}\right) \quad \forall(x, \lambda) \in M(\lambda), n=1,2, \ldots
$$

Taking the limit as $n \rightarrow \infty$, we have

$$
\lim _{n \rightarrow \infty} \tau\left(\bar{x}, w^{(n)}, \bar{\lambda}, u, \eta, \lambda^{t_{n}}\right) \leq \lim _{n \rightarrow \infty} \tau\left(x, w^{(n)}, \lambda, \hat{u}, \hat{\eta}, \lambda^{t_{n}}\right) \quad \forall(x, \lambda) \in M(\lambda) .
$$

From the finiteness of the sum, we get

$$
\tau\left(\bar{x}, \lim _{n \rightarrow \infty} w^{(n)}, \bar{\lambda}, u, \eta, \lim _{n \rightarrow \infty} \lambda^{t_{n}}\right) \leq \tau\left(x, \lim _{n \rightarrow \infty} w^{(n)}, \lambda, \hat{u}, \hat{\eta}, \lim _{n \rightarrow \infty} \lambda^{t_{n}}\right), \quad \forall(x, \lambda) \in M(\lambda),
$$

therefore,

$$
\tau\left(\bar{x}, \bar{w}, \bar{\lambda}, u, \eta, \bar{\lambda}^{t}\right) \leq \tau\left(x, w, \lambda, \hat{u}, \hat{\eta}, \bar{\lambda}^{t}\right) \quad \forall(x, \lambda) \in M(\lambda)
$$

that is, $\left(\bar{w}, \bar{\lambda}^{t}\right) \in T(\bar{x}, \bar{\lambda})$, and hence the set $T(\bar{x}, \bar{\lambda})$ is closed. 
TheOREM 3.3. For a certain degree of $\alpha$, the set $T(\bar{x}, \bar{\lambda})$ is a cone with vertex at the origin. Proof. It is clear that $\left(w, \lambda^{t}\right)=(0,0) \in T(\bar{x}, \bar{\lambda})$. Suppose that $\left(\tilde{w}, \tilde{\lambda}^{t}\right) \in T(\bar{x}, \bar{\lambda})$, then

$$
\tau\left(\bar{x}, \tilde{w}, \bar{\lambda}, u, \eta, \tilde{\lambda}^{t}\right) \leq \tau\left(x, \tilde{w}, \lambda, u, \eta, \tilde{\lambda}^{t}\right) \quad \forall(x, \lambda) \in M(\lambda)
$$

therefore,

$$
\tau\left(\bar{x}, \gamma \tilde{w}, \bar{\lambda}, u, \eta, \gamma \tilde{\lambda}^{t}\right) \leq \tau\left(x, \gamma \tilde{w}, \lambda, u, \eta, \gamma \tilde{\lambda}^{t}\right) \quad \forall(x, \lambda) \in M(\lambda)
$$

and $\gamma \geq 0$, that is, $\left(\gamma \tilde{w}, \gamma \tilde{\lambda}^{t}\right) \in T(\bar{x}, \bar{\lambda})$ for all $\gamma \geq 0$, hence the result follows.

LEMma 3.4. For a certain degree of $\alpha$, if problem (3.5)-(3.6) is stable for all $\left(w, \lambda^{t}\right)$ such that $M(\lambda) \neq \phi$, then $\bar{\alpha}=\mu_{\tilde{\lambda}}\left(\bar{\lambda}_{i}\right),\left(w, \lambda^{t}\right) \in T(\bar{x}, \bar{\lambda})$.

Proof. If $T(\bar{x}, \bar{\lambda})=\left\{\left(\bar{w}, \bar{\lambda}^{t}\right)\right\}$, then the result is clear. Assume that $\left(\widehat{w}, \hat{\lambda}^{t}\right) \in T(\bar{w}, \bar{\lambda}),\left(\widehat{w}, \hat{\lambda}^{t}\right)$ $\neq\left(\bar{w}, \bar{\lambda}^{t}\right)$, then by the assumption and from the KTSP necessary optimality theorem [6], it follows that $(\bar{x}, \bar{\lambda})$ and some $\hat{\xi} \in \mathbb{R}^{m}, \hat{\xi} \geq 0$ solve KTSP and $\hat{\xi}_{i}\left[\alpha-\mu_{\tilde{\lambda}_{i}}\left(\hat{\lambda}^{t}\right)\right]=0$, that is, for all $(x, \lambda) \in M(\lambda)$, and for all $u \geq 0, \xi \geq 0$, we have

$$
\tau\left(\bar{x}, \bar{w}, \bar{\lambda}, u, \xi, \hat{\lambda}^{t}\right) \leq \tau\left(\bar{x}, \bar{w}, \bar{\lambda}, \hat{u}, \hat{\xi}, \hat{\lambda}^{t}\right) \leq \tau\left(x, w, \lambda, \hat{u}, \hat{\xi}, \hat{\lambda}^{t}\right)
$$

and $\hat{u} g(\bar{x})=0, \tau\left(x, w, \lambda, u, \xi, v^{t}\right)=\sum_{i=1}^{m} w_{i} f_{i}(x, \lambda)+\sum_{j=1}^{k} u_{j} g_{j}(x)-\sum_{i=1}^{k} \xi_{i}\left(\alpha-\mu_{\tilde{\lambda}_{i}}\left(\lambda_{i}^{t}\right)\right)$ since $\hat{u} g(\bar{x})=0$ and $\hat{\xi}_{\tilde{\lambda}}\left(\hat{\lambda}^{t}\right)=\hat{\xi} \bar{\alpha}$ for certain degree of $\alpha=\bar{\alpha}$. Then

$$
\tau\left(\bar{x}, \bar{w}, \bar{\lambda}, u, \xi, \hat{\lambda}^{t}\right) \leq \sum_{i=1}^{m} \overline{w_{i}} f_{i}(\bar{x}, \bar{\lambda}) \leq \tau\left(x, w, \lambda, \hat{u}, \hat{\xi}, \hat{\lambda}^{t}\right),
$$

from which it follows that

$$
\tau\left(\bar{x}, \tilde{w}, \bar{\lambda}, u, \xi, \hat{\lambda}^{t}\right) \leq \tau\left(\bar{x}, \widehat{w}, \bar{\lambda}, \hat{u}, \widehat{\xi}, \hat{\lambda}^{t}\right) \leq \tau\left(x, w, \lambda, \hat{u}, \widehat{\xi}, \hat{\lambda}^{t}\right)
$$

therefore from the KTSP sufficient optimality theorem, it follows that $\mu_{\tilde{\lambda}}\left(\hat{\lambda}^{t}\right)=\bar{\alpha},\left(\widehat{w}, \hat{\lambda}^{t}\right) \in$ $T(\bar{x}, \bar{\lambda})$.

Theorem 3.5. For a certain degree of $\alpha$, if the problem (3.5)-(3.6) is stable for all $\left(w, \lambda^{t}\right)$ such that $M(\lambda) \neq \phi$, then the set $T(\bar{x}, \bar{\lambda})$ is star shaped with point of common visibility $\mu_{\tilde{\lambda}}\left(\bar{\lambda}^{t}\right)=\alpha$ and closed.

Proof. From the previous lemma, it follows that $\left(\bar{w}, \bar{\lambda}^{t}\right) \in T(\bar{x}, \bar{\lambda})$. Let another point $(\widetilde{w}$, $\left.\tilde{\lambda}^{t}\right) \in T(\bar{x}, \bar{\lambda})$, then by the assumption and from the KTSP necessary optimality theorem, it follows that $(\bar{x}, \bar{\lambda})$ and some $\tilde{u} \geq 0, \tilde{\xi} \geq 0$ solve KTSP and $\tilde{u} g(\bar{x})=0, \tilde{\xi}\left[\bar{\alpha}-\mu_{\tilde{\lambda}}\left(\bar{\lambda}^{t}\right)\right]=0$ for certain degree of $\alpha=\bar{\alpha}$, then for all $(x, \lambda) \in M(\lambda)$ and for all $u \geq 0, \xi \geq 0$, we have

$$
\tau\left(\bar{x}, \bar{w}, \bar{\lambda}, u, \xi, \bar{\lambda}^{t}\right) \leq \tau\left(\bar{x}, \bar{w}, \bar{\lambda}, \tilde{u}, \tilde{\xi}, \bar{\lambda}^{t}\right) \leq \tau\left(x, w, \lambda, \tilde{u}, \tilde{\xi}, \bar{\lambda}^{t}\right),
$$

$\tilde{u}_{j} g_{j}(\bar{x})=0, \tilde{\xi}_{i}\left[\bar{\alpha}-\mu_{\tilde{\lambda}_{i}}\left(\bar{\lambda}_{i}^{t}\right)\right]=0$ for certain degree of $\alpha=\bar{\alpha}$ 
Putting $u_{j}=\gamma U_{j}, \xi_{r}=\gamma \zeta_{r}, w_{i}=\gamma W_{i}, \gamma \geq 0$, and $(1-\gamma) \widetilde{u}_{j} g_{j}(\bar{x})=0,(1-\gamma) \tilde{\xi}_{r}[\bar{\alpha}-$ $\left.\mu_{\tilde{\lambda}}\left(\bar{\lambda}^{t}\right)\right]=0$, we deduce from the relation (3.20) and for a certain degree of $\alpha=\bar{\alpha}$ that

$$
\begin{aligned}
& \sum_{i=1}^{m} \bar{w}_{i} f_{i}(\bar{x}, \bar{\lambda})+\sum_{j=1}^{k} u_{j} g_{j}(\bar{x})-\sum_{i=1}^{m} \xi_{i}\left[\mu_{\tilde{\lambda}_{i}}\left(\bar{\lambda}_{i}^{t}\right)-\bar{\alpha}\right] \\
& \leq \sum_{i=1}^{m} \bar{w}_{i} f_{i}(\bar{x}, \bar{\lambda})+\sum_{j=1}^{k} \tilde{u}_{j} g_{j}(\bar{x})-\sum_{i=1}^{m} \tilde{\xi}_{i}\left[\mu_{\tilde{\lambda}_{i}}\left(\bar{\lambda}_{i}^{t}\right)-\bar{\alpha}\right] \\
& \leq \sum_{i=1}^{m} w_{i} f_{i}(x, \lambda)+\sum_{j=1}^{k} \tilde{u}_{j} g_{j}(x)-\sum_{i=1}^{m} \tilde{\xi}_{i}\left[\mu_{\tilde{\lambda}_{i}}\left(\bar{\lambda}_{i}^{t}\right)-\bar{\alpha}\right] .
\end{aligned}
$$

Then,

$$
\begin{aligned}
\sum_{i=1}^{m} \bar{W}_{i} & {\left[f_{i}(\bar{x}, \bar{\lambda})-(1-\gamma) f_{i}(\bar{x}, \bar{\lambda})\right]+\sum_{j=1}^{k} \tilde{U}_{j}\left[g_{j}(\bar{x})-(1-\gamma) g_{j}(\bar{x})\right] } \\
& -\sum_{i=1}^{m} \zeta_{i}\left[\mu_{\tilde{\lambda}_{i}}\left(\bar{\lambda}_{i}^{t}\right)-\alpha-(1-\gamma)\left(\mu_{\tilde{\lambda}_{i}}\left(\bar{\lambda}_{i}^{t}\right)-\alpha\right)\right] \\
\leq & \sum_{i=1}^{m} \bar{w}_{i} f_{i}\left(\bar{x}, \bar{\lambda}_{i}\right)+\sum_{j=1}^{k} \tilde{u}_{j} g_{j}(\bar{x})-\sum_{i=1}^{m} \tilde{\xi}_{i}\left[\mu_{\tilde{\lambda}_{i}}\left(\bar{\lambda}^{t}\right)-\alpha\right] \\
\leq & \sum_{i=1}^{m} W_{i}\left[f_{i}\left(x, \lambda_{i}\right)-(1-\gamma) f_{i}\left(x, \lambda_{i}\right)\right]+\sum_{j=1}^{k} \tilde{U}_{j}\left[g_{j}(x)-(1-\gamma) g_{j}(\bar{x})\right] \\
& -\sum_{i=1}^{m} \tilde{\xi}_{i}\left[\left(\mu_{\tilde{\lambda}_{i}}\left(\bar{\lambda}_{i}^{t}\right)-\alpha\right)-(1-\gamma)\left(\mu_{\tilde{\lambda}_{i}}\left(\bar{\lambda}_{i}^{t}\right)-\alpha\right)\right] \quad \forall U \geq 0, \xi \geq 0, \gamma \geq 0 .
\end{aligned}
$$

Therefore, it follows from the KTSP sufficient optimality theorem and from the above relation that $(1-\gamma) w_{i} f_{i}(\bar{x}, \bar{\lambda}),(1-\gamma) g_{j}(\bar{x})$, and $\gamma \bar{\alpha}-(1-\gamma) \mu_{\tilde{\lambda}}(\bar{\lambda})$ belong to $T(\bar{x}, \bar{\lambda})$ for all $\gamma \geq 0$. Hence the set $T(\bar{x}, \bar{\lambda})$ is star shaped with point of common visibility $\left(\bar{w}, \bar{\lambda}^{t}\right)$. If the set $T(\bar{x}, \bar{\lambda})$ is a one-point set or the whole space, then it is clearly closed. Choose a sequence of points $\left(w^{(n)}, \lambda^{t_{n}}\right) \in T(\bar{x}, \bar{\lambda})$ which is convergent to $\left(w, \lambda^{t}\right)$, that is, $\lim _{n \rightarrow \infty}\left(w^{(n)}, \lambda^{t_{n}}\right)=$ $\left(w, \lambda^{t}\right)$. Then

$$
\sum_{i=1}^{m} w_{i}^{(n)} f_{i}\left(\bar{x}, \bar{\lambda}_{i}\right) \leq \sum_{i=1}^{m} w_{i}^{(n)} f_{i}\left(x, \lambda_{i}\right) \quad \forall g(x) \leq 0, \quad \mu_{\tilde{\lambda}}\left(\lambda^{t_{n}}\right) \geq \alpha, \quad n=1,2, \ldots,
$$

and as $n \rightarrow \infty$, we get

$$
\sum_{i=1}^{m} w_{i} f_{i}\left(\bar{x}, \bar{\lambda}_{i}\right) \leq \sum_{i=1}^{m} w_{i} f_{i}\left(x, \lambda_{i}\right) \quad \forall g(x) \leq 0, \quad \mu_{\tilde{\lambda}}\left(\lambda^{t}\right) \geq \alpha,
$$

that is, $\left(w, \lambda^{t}\right) \in T(\bar{x}, \bar{\lambda})$, and therefore the set $T(\bar{x}, \bar{\lambda})$ is closed.

Theorem 3.6. For a certain degree of $\alpha$, if the problem (3.5)-(3.6) is stable for all $\left(w, \lambda^{t}\right)$ such that $M(\lambda) \neq \phi$, then the set $T(\bar{x}, \bar{\lambda})$ is either one point set or unbounded.

Proof. For a certain degree of $\alpha$, if the set $T(\bar{x}, \bar{\lambda})$ consists only of one point, then it is clear that this point is $\left(\bar{w}, \bar{\lambda}^{t}\right)$. If $T(\bar{x}, \bar{\lambda})$ contains another point $\left(w^{1}, \lambda^{t_{1}}\right)$, then it is clear from relation (3.20) and from the KTSP sufficient optimality theorem that

$$
\left\{\left(w, \lambda^{t}\right) \in \mathbb{R}^{4 m}:(1-\gamma) w^{\prime} f(\bar{x}, \bar{\lambda}),(1-\gamma) g(\bar{x}),(1-\gamma) \mu_{\tilde{\lambda}}\left(\lambda^{t^{\prime}}\right)-\gamma \alpha\right\} \subseteq T(\bar{x}, \bar{\lambda}),
$$

$\gamma \geq 0$, that is, the set $T(\bar{x}, \bar{\lambda})$ is unbounded. 


\section{Conclusion}

The stability set of the first kind for fuzzy parametric multiobjective nonlinear programming, which represents the set of all fuzzy parameters for which an $\alpha$-Pareto optimal point for one fuzzy parameter rests $\alpha$-Pareto optimal for all fuzzy parameters, has been analyzed qualitatively in the author's notes $[2,3,4]$, where all the functions are assumed to posses the first-order partial derivation on $\mathbb{R}^{n}$. In this note, no differentiability assumptions are needed and the KTSP necessary and sufficient optimality theorems are used in the derivation of the proposed results.

Future extension to this work is the characterization of the stability set of the first kind for MONLP with fuzzy parameters in both the objective functions and the constraints without any differentiability assumptions. Another field of extension is the field of fuzzy parametric nonconvex MONLP, where more difficulties may be found in the characterization of the stability set of the first kind of such problems.

\section{References}

[1] D. Dubois and H. Prade, Fuzzy Sets and Systems. Theory and Applications, Mathematics in Science and Engineering, vol. 144, Academic Press, New York, 1980.

[2] M. A. Kassem, Interactive stability of multiobjective nonlinear programming problems with fuzzy parameters in the constraints, Fuzzy Sets and Systems 73 (1995), no. 2, 235-243.

[3] _ Decomposition of the fuzzy parametric space in multiobjective nonlinear programming problems, European J. Oper. Res. 101 (1997), 204-219.

[4] M. A. Kassem and E. I. Ammar, Stability of multiobjective nonlinear programming problems with fuzzy parameters in the constraints, Fuzzy Sets and Systems 74 (1995), no. 3, 343-351.

[5] A. Kaufmann and M. M. Gupta, Fuzzy Mathematical Models in Engineering and Management Science, North-Holland, Amsterdam, 1988.

[6] O. L. Mangasarian, Nonlinear Programming, McGraw-Hill, New York, 1969.

[7] S. A. Orlovski, Multiobjective programming problems with fuzzy parameters, Control Cybernet. 13 (1984), no. 3, 175-183.

[8] M. S. Osman and A.-Z. H. El-Banna, Stability of multiobjective nonlinear programming problems with fuzzy parameters, Math. Comput. Simulation 35 (1993), no. 4, 321-326.

[9] M. Sakawa and H. Yano, Interactive decision making for multiobjective nonlinear programming problems with fuzzy parameters, Fuzzy Sets and Systems 29 (1989), no. 3, 315-326.

[10] H. Tanaka and K. Asai, Fuzzy linear programming problems with fuzzy numbers, Fuzzy Sets and Systems 13 (1984), no. 1, 1-10.

Mohamed Abd El-Hady Kassem: Department of Mathematics, Faculty of Science, Tanta University, 31527 Tanta, Egypt

E-mail address: mohd60_371@hotmail.com 


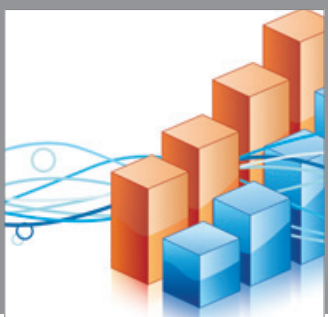

Advances in

Operations Research

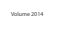

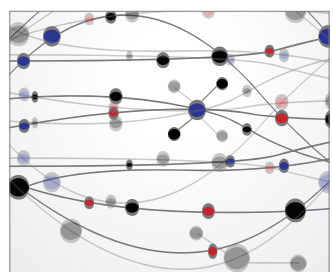

\section{The Scientific} World Journal
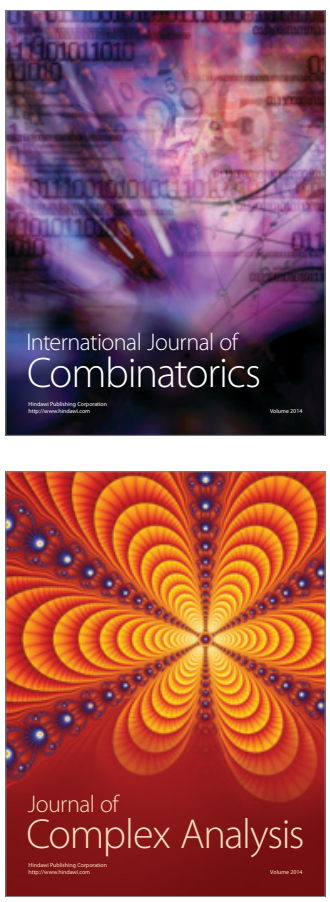

International Journal of

Mathematics and

Mathematical

Sciences
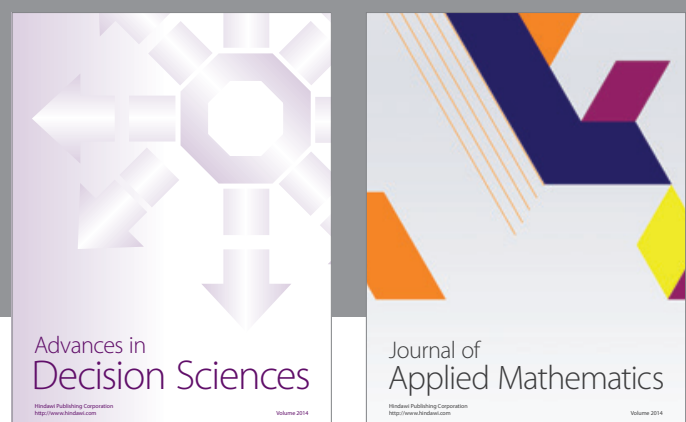

Journal of

Applied Mathematics
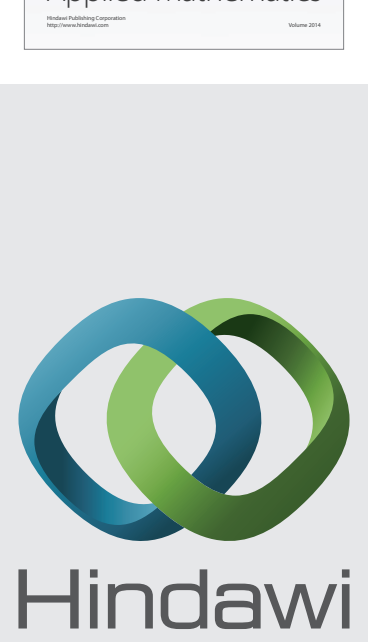

Submit your manuscripts at http://www.hindawi.com
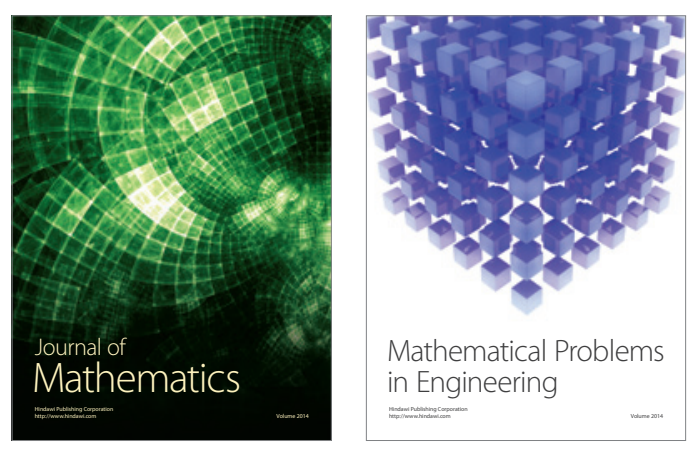

Mathematical Problems in Engineering
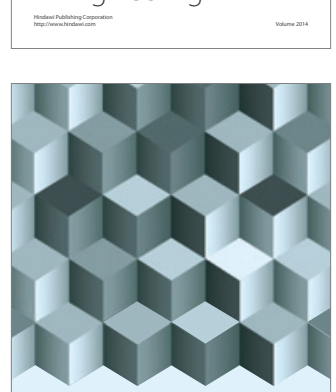

Journal of

Function Spaces
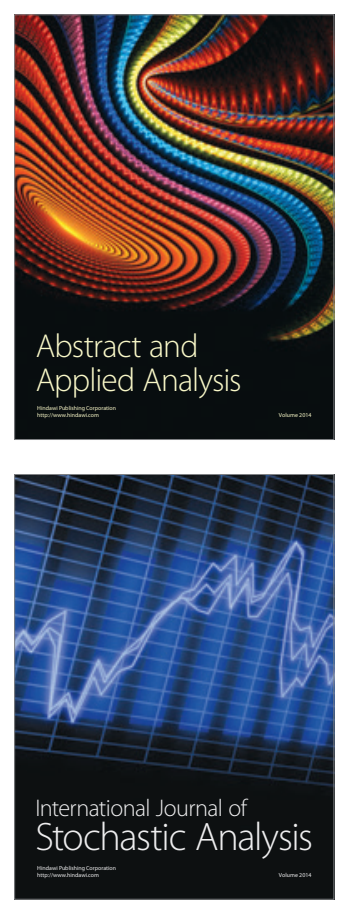

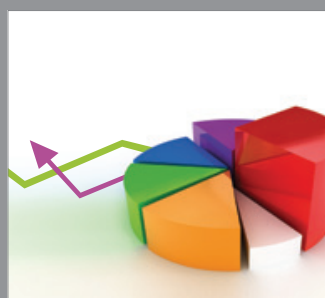

ournal of

Probability and Statistics

Promensencen
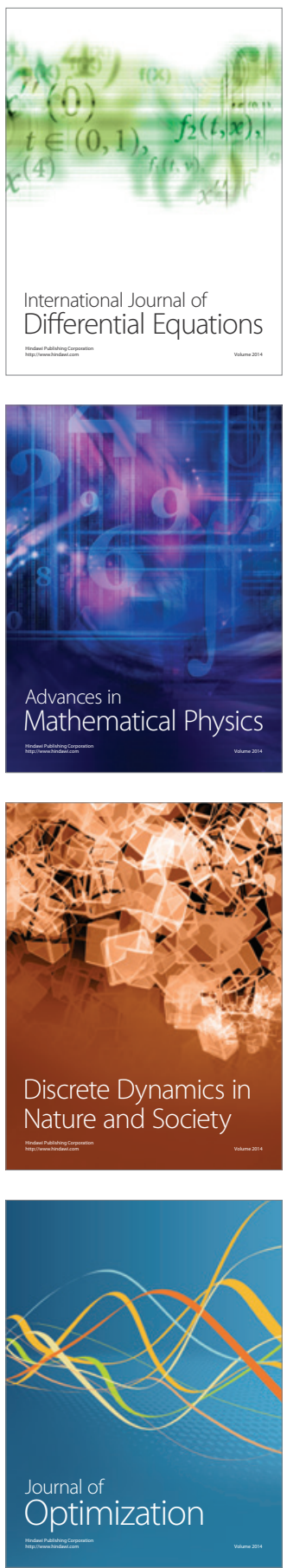